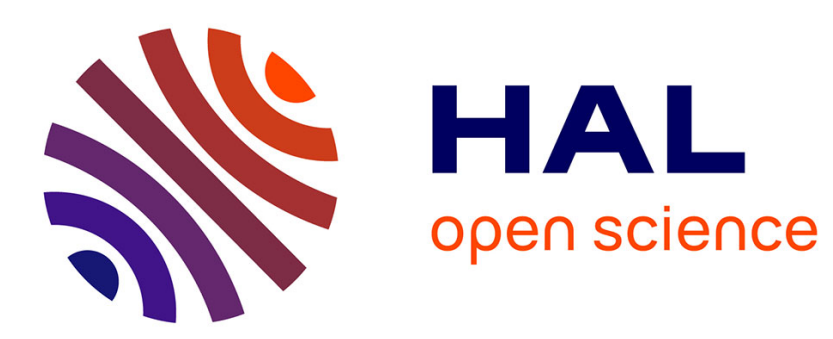

\title{
Modular Active Curiosity-Driven Discovery of Tool Use
}

Sébastien Forestier, Pierre-Yves Oudeyer

\section{To cite this version:}

Sébastien Forestier, Pierre-Yves Oudeyer. Modular Active Curiosity-Driven Discovery of Tool Use. IEEE/RSJ International Conference on Intelligent Robots and Systems, 2016, Daejeon, South Korea. hal-01384566

\section{HAL Id: hal-01384566 https://hal.science/hal-01384566}

Submitted on 20 Oct 2016

HAL is a multi-disciplinary open access archive for the deposit and dissemination of scientific research documents, whether they are published or not. The documents may come from teaching and research institutions in France or abroad, or from public or private research centers.
L'archive ouverte pluridisciplinaire HAL, est destinée au dépôt et à la diffusion de documents scientifiques de niveau recherche, publiés ou non, émanant des établissements d'enseignement et de recherche français ou étrangers, des laboratoires publics ou privés. 


\title{
Modular Active Curiosity-Driven Discovery of Tool Use
}

\author{
Sébastien Forestier ${ }^{1}$ and Pierre-Yves Oudeyer ${ }^{2}$
}

\begin{abstract}
This article studies algorithms used by a learner to explore high-dimensional structured sensorimotor spaces such as in tool use discovery. In particular, we consider goal babbling architectures that were designed to explore and learn solutions to fields of sensorimotor problems, i.e. to acquire inverse models mapping a space of parameterized sensorimotor problems/effects to a corresponding space of parameterized motor primitives. However, so far these architectures have not been used in high-dimensional spaces of effects. Here, we show the limits of existing goal babbling architectures for efficient exploration in such spaces, and introduce a novel exploration architecture called Model Babbling (MB). MB exploits efficiently a modular representation of the space of parameterized problems/effects. We also study an active version of Model Babbling (the MACOB architecture). These architectures are compared in a simulated experimental setup with an arm that can discover and learn how to move objects using two tools with different properties, embedding structured high-dimensional continuous motor and sensory spaces.
\end{abstract}

\section{INTRODUCTION}

A major challenge in robotics is to learn sensorimotor models in high-dimensional continuous motor and perceptual spaces. Of particular interest is the acquisition of inverse models which map a space of sensorimotor problems to a space of motor programs that solve them. For example, this could be a robot learning which movements of the arm and hand can push or throw an object in each of several target locations, or which arm movements allow to produce which displacements of several objects potentially interacting with each other, e.g. in the case of tool use. Specifically, acquiring such repertoires of skills through incremental exploration of the environment has been argued to be a key target for lifelong developmental learning [1], [2], [3].

To approach this challenge, various works have considered the parameterization of these motor and problem spaces. For example, motor programs can be encoded through Dynamical Movement Primitives parameterized by a vector of real numbers [4]. Similarly, it is possible to embed targeted sensorimotor problems (also called space of effects or task space) in a dual parameterized space such as the coordinates of the target object location [5], [6], [7], potentially combined with parameters characterizing the position of obstacles [4].

This dual parameterization is useful for several reasons. First, given a database of experiences associating parameters of motor programs to a set of sensorimotor problems

\footnotetext{
${ }^{1}$ Sébastien Forestier is with Inria Bordeaux Sud-Ouest and Université Bordeaux, 200 Avenue de la Vieille Tour, 33405 Talence, France sebastien. forestierdinria.fr

${ }^{2}$ Pierre-Yves Oudeyer is with Inria Bordeaux Sud-Ouest and Ensta ParisTech, 200 Avenue de la Vieille Tour, 33405 Talence, France pierre-yves. oudeyerdinria.fr
}

they solve (e.g. the effects they produce), it is possible to use optimization and regression techniques to infer the parameters of motor programs that solve new sensorimotor problems which parameters were not encountered during training [4], [5], [6], [7], [8]. Second, it allows efficient data collection leveraging the interactions among sensorimotor problems as achieved in goal babbling exploration [7], [9], [10] and other related approaches [8]: when the learner is searching for parameters optimizing one sensorimotor problem (typically using policy search or related stochastic optimization methods [11]), it will often discover parameters that are improving other sensorimotor problems - and update their current best solutions accordingly [7].

Next to approaches that have considered finite sets of parameterized problems [4], [12], other approaches [7], [8], [9], [10], [13] have considered the challenge of autonomous exploration and learning of continuous fields of parameterized problems (e.g. discovering and learning all the feasible displacements of objects and their motor solutions). Among them, the technique of goal babbling [7], [9], [10] (which can be made active [7], [10]) was shown to be highly efficient for complex tasks such as learning to throw an object in all direction with a flexible fishing rod [14], learning omnidirectional legged locomotion on slipping surfaces [7] or learning to control a robotic pneumatic elephant trunk [9].

However, to our knowledge, results of goal babbling approaches as well as results of other approaches to learning inverse models were so far achieved in relatively lowdimensional spaces of parameterized problems. Furthermore, they were also experimented in sensorimotor spaces with little structure, and in particular have not yet been applied to sensorimotor problems involving tool use.

In this article, the primary question we address is: Can goal babbling approaches efficiently drive exploration in high-dimensional structured sensorimotor spaces, such as in tool use discovery? As we will show, applying them as they exist does not allow an efficient exploration of the sensorimotor space. Rather, we will present a novel algorithmic architecture for exploration, called Model Babbling, that drives sensorimotor data collection by considering a modular representation of the sensorimotor space: instead of considering a flat architecture mapping a motor space to a single high-dimensional space of effects, it considers a set of submodels mapping the motor space to various subspaces of the space of effects. When selected, each of these submodels is explored using the goal babbling approach, and the architecture leverages the fact that exploring one submodel produces data that can improve other submodels.

A secondary issue we study is whether active learning 
methods can improve the efficiency of this Model Babbling approach. In particular, we present the Modular Active Curiosity-driven mOdel Babbling (MACOB) architecture, where a measure of empirical learning progress is used by a multi-armed bandit algorithm to select which model to explore [2], [7], [10]. This curiosity-driven exploration algorithm can be related to work using intrinsic motivations in the Reinforcement Learning literature [2], [15], [16].

The study we present is instantiated in a simulated experimental setup 11 with an arm that can discover and learn how to move objects using two tools with different properties. Compared to other work that have studied autonomous tool use learning [17], [18], [19], [20], this study is original in that it combines 1) considering the problem of how to design efficient exploration algorithms rather than how to design efficient exploitation algorithms that can build compact models from the data collected through exploration; 2) considering a problem of tool use discovery where tools are objects with initially no special status with respect to other objects, i.e. the robot does not know they are "tools".

\section{Methods}

The problem settings for the learning agent is to explore its sensorimotor space and collect data so as to generate a diversity of effects and that this collected database of learning exemplars can be used to build inverse models to be able to reproduce those effects. An agent is described as two independent components: an exploration algorithm and an exploitation algorithm (see Fig. 11). The exploration algorithm decides at each iteration which motor command $m$ to explore, and gathers a sensory feedback $s$ to update a database of sensorimotor experiences. We suppose that the motor space $M$ and the sensory space $S$ are continuous and high-dimensional, and that a factorization of $S$ as a product of sensory subspaces that represents the items of the environment can be given to the agent. As detailed below, the exploration algorithm can make use of the current database of sensorimotor experiences to define a coarse but fast surrogate inverse model to orient the exploration process. On the other hand, the exploitation algorithm uses the database built during exploration to generate a potentially more precise inverse model, i.e. to find motor commands to reach sensory goals given by the experimenter based on the explored data. The inverse model of the exploitation algorithm can be built during exploration as an incremental and asynchronous process, or built at the end of exploration as a batch process. Next we describe the exploration architectures, the experimental setup and exploitation architectures.

\section{A. Exploration Architectures}

1) Random Motor Babbling: We first define a control architecture where the agent always chooses random motor commands to try in the environment (RmB, see Algo. 11).

${ }^{1}$ Open-source code, notebooks and videos are available on GitHub at https://github.com/sebastien-forestier/IROS2016

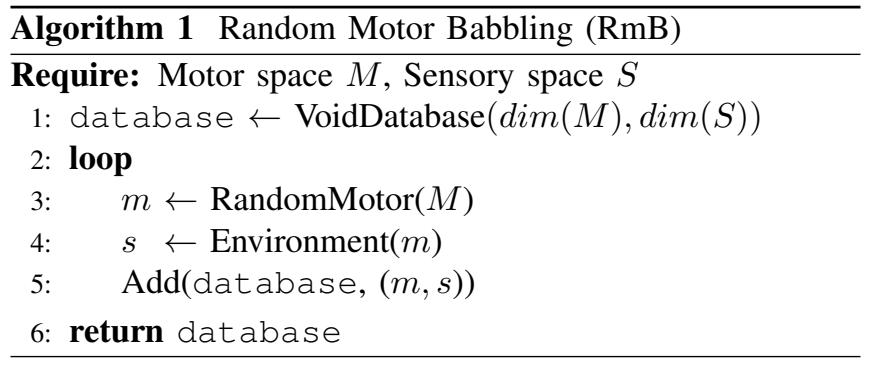

2) Random Goal Babbling: In the following exploration architectures the agent performs Goal Babbling. With this method, it self-generates goals in the sensory space and tries to reach them but adds some exploration noise to its motor commands to discover new effects. To generate those goals, different strategies have been studied [7]. It was shown that estimating the learning progress in different regions of the sensory space and generating the goals where the progress is high leads to fast learning. However, this cannot be applied in a high-dimensional sensory space as a learning progress signal could not be efficiently estimated.

Consequently, we use random goal babbling: goals are randomly generated in the sensory space. This method was nevertheless proven to be highly efficient in complex sensorimotor spaces [9]. To perform goal babbling, the agent uses a sensorimotor model that learns a mapping between $M$ and $S$ and provide the inverse inference of a probable motor command $m$ to reach a given sensory goal $s_{g}$ (see Algo. 2 and 3). The sensorimotor model stores sensorimotor information of the form $(m+\eta, s)$ with $m+\eta$ being the inferred motor parameters to reach the sensory goal, plus gaussian exploration noise (of standard deviation $\sigma=$ 0.01 ), and $s \in S$ the associated sensory feedback in a sensorimotor database. Section II-A.5 explains in more detail the two different algorithms that will be used to implement inverse models. We use the Explauto autonomous exploration library [21] to implement the sensorimotor models and goal babbling. In our implementation, the agent first begins by exploring random motor commands to bootstrap the sensorimotor model until at least 2 distinct sensory points have been reached, and then it starts goal babbling.

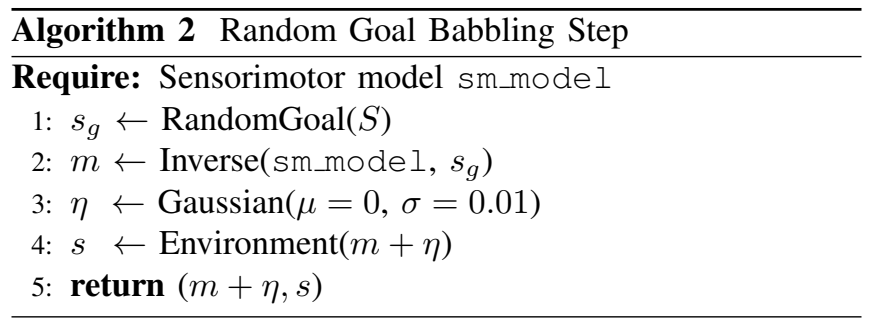

3) Model Babbling: We call flat exploration architecture the random goal babbling strategy applied to explore directly a mapping between the motor space $M$ and the sensory space $S$. However, the high-dimensional sensory space (e.g. 93D, see experimental setup after) can be separated into several subspaces to reflect the perception of the different items of 


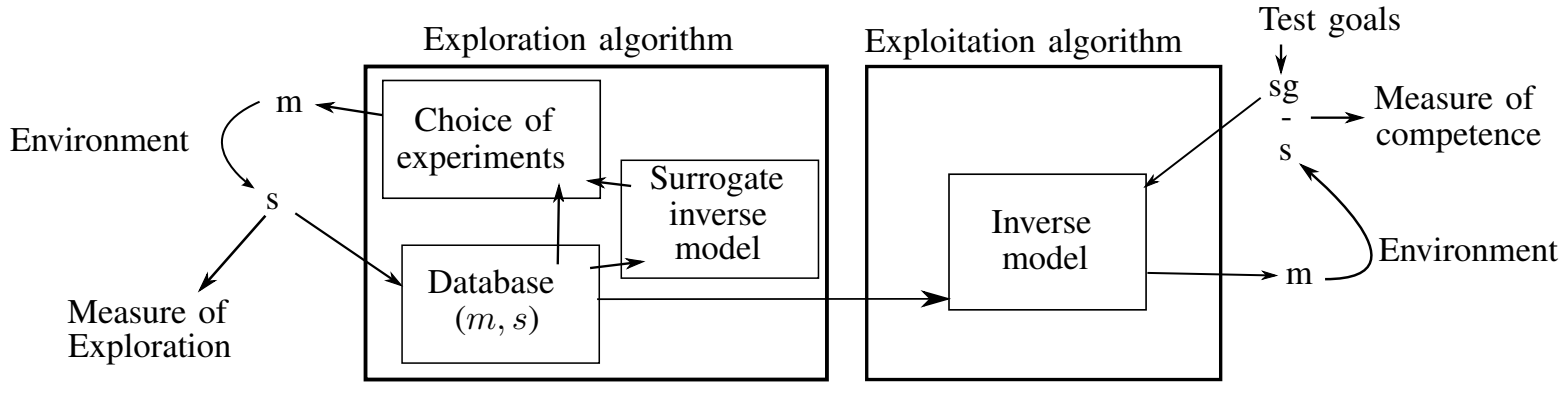

Fig. 1. Agent's two components: the exploration and exploitation algorithms.

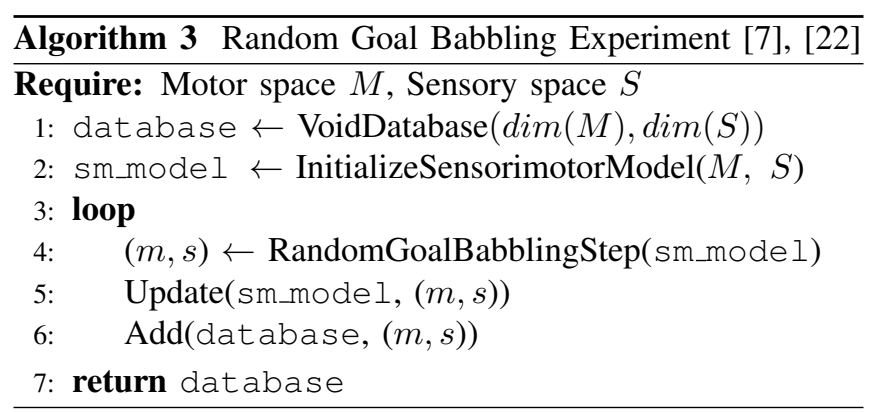

the environment (e.g. $p=15$ subspaces). We thus define a modular architecture that explores $p$ sensorimotor models at the same time (one model for each sensory subspace). Each of those modules functions in the same way as a random goal babbling flat architecture, with $M$ as motor space but a specific sensory subspace. However, at each iteration the modular architecture first has to choose the module that will perform goal babbling - pick a random goal in the corresponding sensory subspace. We call this procedure Model Babbling. In a first condition, the babbling module is randomly chosen, which we call Random Model Babbling (See Algo. 4). Once a model is chosen, the agent generates a random goal in the sensory subspace corresponding to that model, infers motor parameters to reach that goal, and adds exploration noise as in the flat architectures. Finally, when motor parameters $m$ have been executed and feedback $s$ received from the environment, the sensorimotor mappings of all modules are updated with their respective part of $s$.

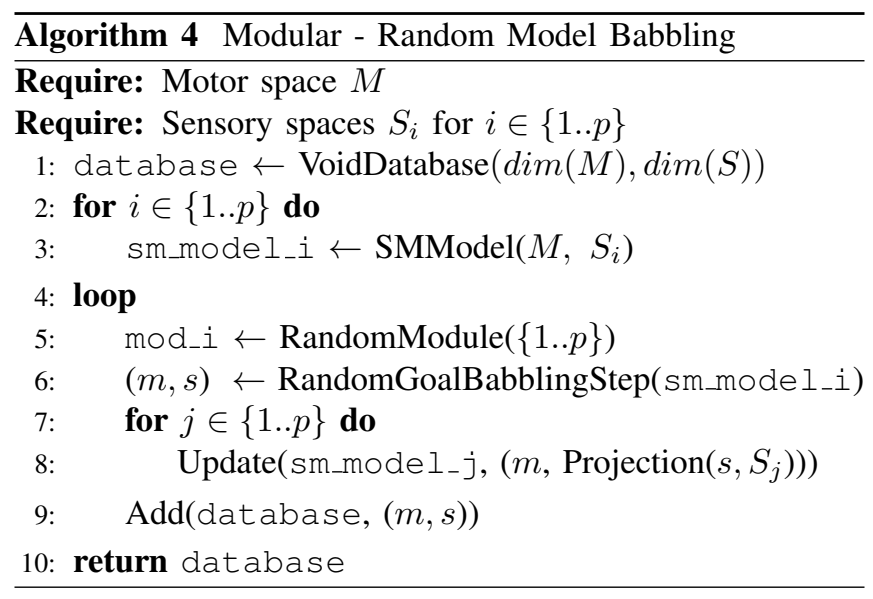

4) Active Model Babbling: In strategic learning, different parameterized problems and strategies to solve them are available and the agent learns which strategies are useful for which problems. It was shown in [23] that an active choice of the outcomes and strategies based on the learning progress on each of them increases learning efficiency compared to a random choice. Also, in [7], the authors develop the SAGGRIAC architecture of algorithms where the sensory space is automatically splitted into regions where the learning progress is monitored, and goals are generated in regions where the progress is high. Here, instead of differentiating the learning progress in different regions of a single space, we differentiate it in different sensory spaces.

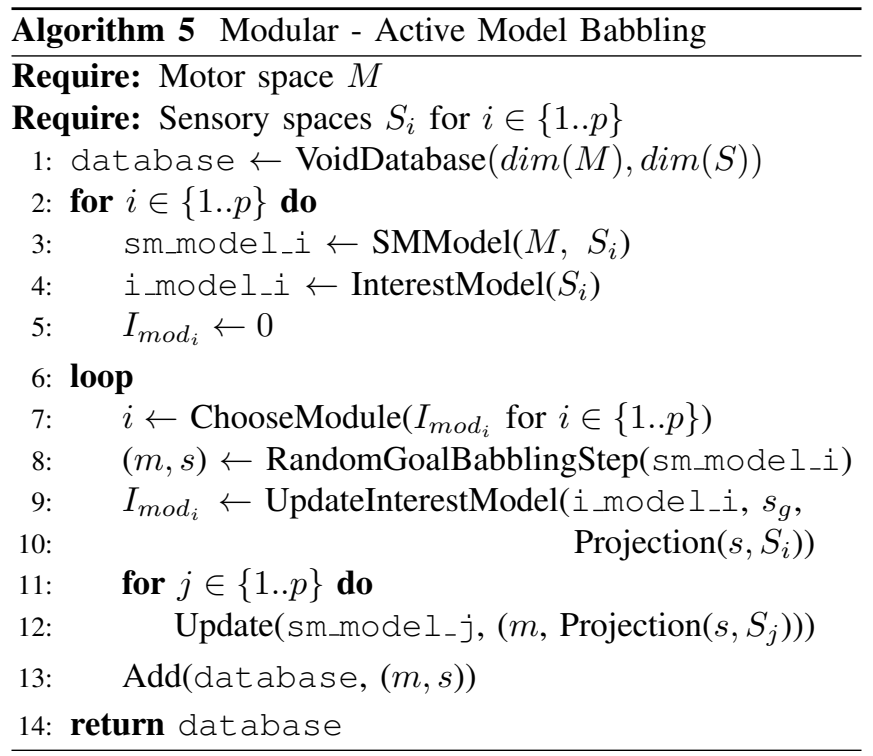

To implement an active choice of model to explore (Active Model Babbling, see Algo. 5), we first define a measure of interest based on the learning progress of each of the $p$ modules (see Algo. 6). When a module has been chosen to babble, it draws a random goal $s_{g}$ and finds motor parameters $m$ to reach this goal. The actually reached outcome $s$ in its sensory subspace might be very different from $s_{g}$. To measure the progress made to reach $s_{g}$, we compare the reached point $s$ with the point $s^{\prime}$ that was reached for the most similar previous goal $s_{g}^{\prime}$. We define a distance $D_{S_{i}}$ between two points $s$ and $s^{\prime}$ in a sensory subspace $S_{i}$ as the 
$L_{2}$ distance divided by the maximal distance in this sensory subspace, in order to scale this measure across subspaces:

$$
D_{S_{i}}\left(s, s^{\prime}\right)=\frac{\left\|s-s^{\prime}\right\|}{\max _{s_{1}, s_{2}}\left\|s_{1}-s_{2}\right\|}
$$

We define the interest $I\left(s_{g}\right)$ associated to the goal $s_{g} \in S_{i}$ :

$$
I\left(s_{g}\right)=\left|D_{S_{i}}\left(s_{g}, s^{\prime}\right)-D_{S_{i}}\left(s_{g}, s\right)\right|
$$

where $s_{g}$ and $s$ are the current goal and reached sensory points, and $s_{g}^{\prime}$ and $s^{\prime}$ are the previous goal of that module that is the closest to $s_{g}$, and its associated reached sensory point. The interest of a module is initialized at 0 and updated to follow the progress of its goals (with rate $n=1000$ ):

$$
I_{\text {mod }}(t)=\frac{n-1}{n} I_{\text {mod }}(t-1)+\frac{1}{n} I\left(s_{g}\right)
$$

where $t$ is the current iteration: $t \in[1 . .100000]$.

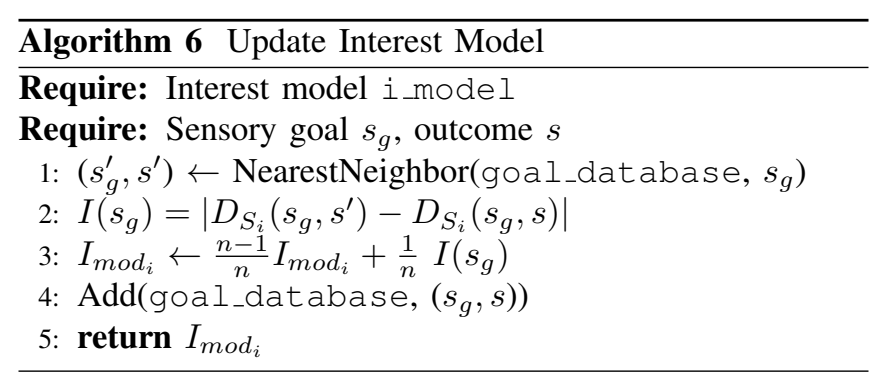

Finally, we implement a multi-armed bandit algorithm to choose the babbling module at each iteration [7], [10]. The choice of module is probabilistic and proportional to their interest, with $\epsilon=10 \%$ of random choice to set up an exploration/exploitation tradeoff. We call MACOB those modular active exploration architectures (Modular Active Curiosity-driven mOdel Babbling).

5) Sensorimotor models: Here we describe two algorithms to provide fast, incremental and online forward and inverse model based on a sensorimotor database of motor commands and associated sensory feedback. The first algorithm is the Nearest Neighbor (NN) algorithm, which finds the nearest neighbor of a given point in a database based on a kd-tree search. The forward model is implemented by the following: given a motor command $m$, the $\mathrm{NN}$ algorithm finds the nearest motor command $m^{\prime}$ in the motor part of the database, and returns the sensory point associated to $m^{\prime}$. Also, the inverse of a sensory goal $s_{g}$ is computed as the motor part $m^{\prime}$ of the nearest neighbor $s^{\prime}$ of $s_{g}$ in the sensory part of the sensorimotor database (see Algo. 7 .

The second algorithm allows to interpolate and extrapolate the forward model around explored points with the Locally Weighted Linear Regression (LWLR, [24]). Given a motor command $m$, LWLR computes a linear regression of the forward model based on the $k=10$ nearest neighbors of $m$ in the motor part of the database, weighted locally. The weights of the $k$ nearest neighbors of $m$ depends on the distance to $m$ with a gaussian decreasing function of standard deviation $\sigma=0.1$, and LWLR then computes the prediction

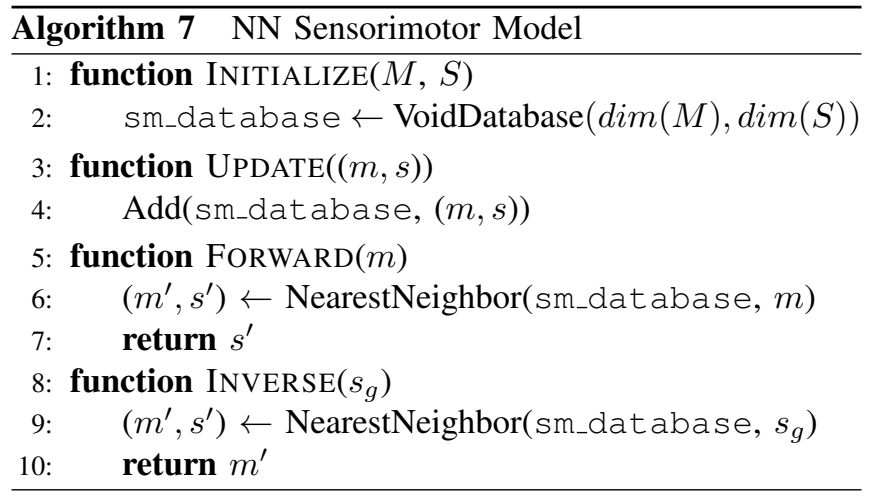

$s_{p}$ of $m$ with this local regression (see Algo. 87. On the other hand, the inverse $m^{*}$ of a sensory goal $s_{g}$ is found by the minimization of the predicted distance between the reached and goal sensory points as the error function $e(m)=$ $\|$ Forward $(m)-s_{g} \|^{2}$ with an optimization algorithm (we use the L-BFGS-B algorithm [25]). We limit the number of forward model evaluations (which uses LWLR) to 200.

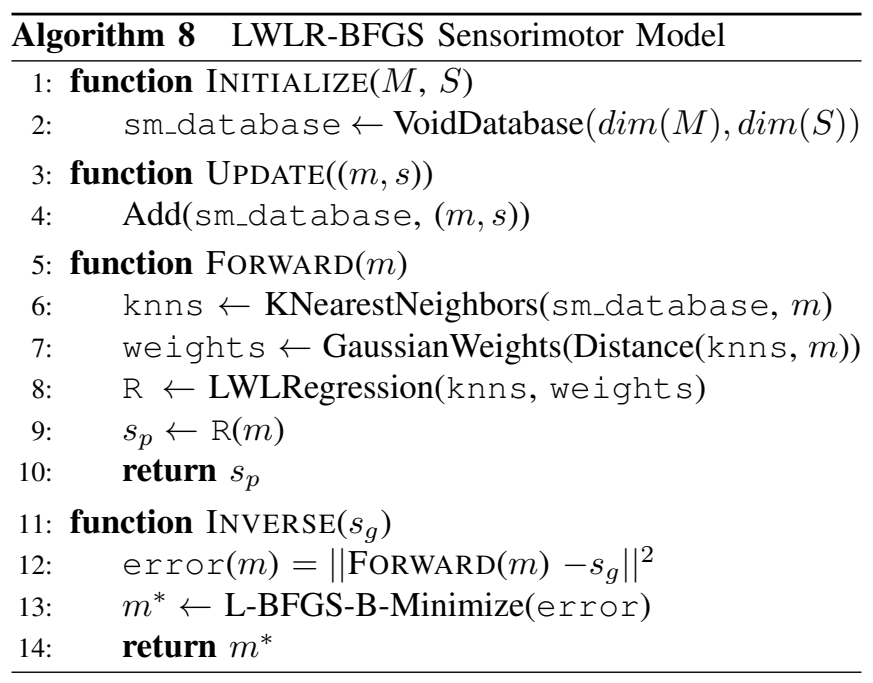

6) Summary of Exploration Architectures:

- RmB: Random motor Babbling control (Algo. 11,

- F-NN-RGB: Flat, Nearest Neighbor forward and inverse models, Random Goal Babbling (Algo. 2, 3, 7),

- F-LWLR-RGB: Flat, Locally Weighted Linear Regression forward model and optimization-based inverse model, Random Goal Babbling (Algo. 2, 3, 8),

- M-NN-RMB: Modular, Nearest Neighbor forward and inverse models, Random Model Babbling (Algo. 2, 3 , 4, 7),

- M-NN-AMB: Modular, Nearest Neighbor forward and inverse models, Learning Progress based Active Model Babbling (Algo. 2, 3, 5, 6, 77,

- M-LWLR-RMB: Modular, Locally Weighted Linear Regression forward model, optimization-based inverse model, Random Model Babbling (Algo. 2, 3, 4, 8,

- M-LWLR-AMB: Modular, Locally Weighted Linear Regression forward model, optimization-based inverse model, Active Model Babbling (Algo. 2, 3, 5, 6, 8). 


\section{B. Experimental setup}

We designed a robotic setup where a 2D simulated arm can grasp two sticks that can be used to move some of the out-of-reach objects (see Fig 2). The different items in the scene and their interactions are described in the next sections.

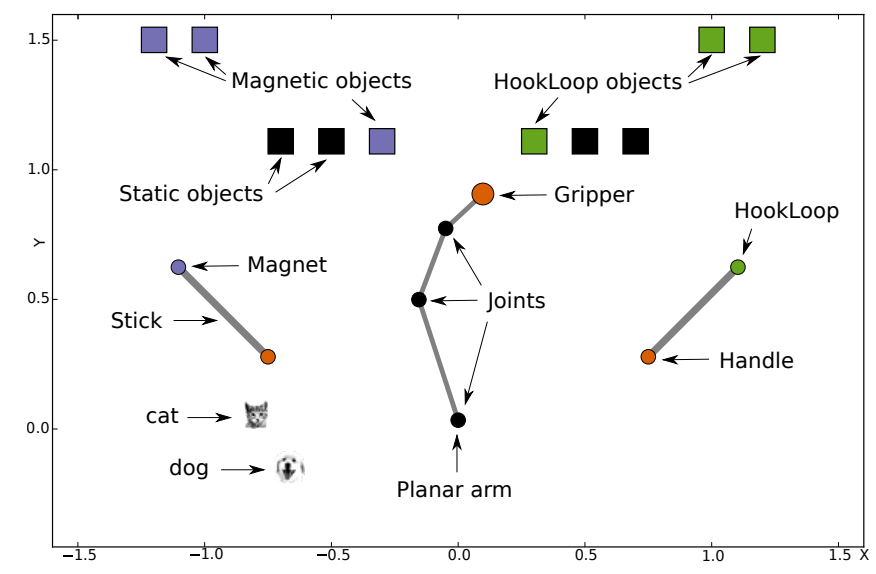

Fig. 2. A possible state of the environment.

1) Robotic Arm: The 2D robotic arm has 3 joints plus a gripper located at the end of the arm. Each joint can rotate from $-\pi \mathrm{rad}$ to $\pi \mathrm{rad}$ around its resting position, mapped to a standard interval of $[-1,1]$. The length of the 3 segments of the arm are $0.5,0.3$ and 0.2 so the length of the arm is 1 unit. The resting position of the arm is vertical with joints at $0 \mathrm{rad}$ and its base is fixed at position $(0,0)$. The gripper $g$ has 2 possible positions: open $(g \geq 0)$ and closed $(g<0)$ and its resting position is open (with $g=0$ ). The robotic arm has 4 degrees of freedom represented by a vector in $[-1,1]^{4}$. A trajectory of the arm is represented as a sequence vectors.

2) Motor Control: We use Dynamical Movement Primitives [26] to control the arm's movement as this framework allows the production of a diversity of arm's trajectories with few parameters. Each of the 4 arm's degrees-of-freedom (DOF) is controlled by a DMP starting at the resting position of the joint. Each DMP is parameterized by one weight on each of 2 basis functions and one weight specifying the end position of the movement. The weights are bounded in the interval $[-1,1]$ and allow each joint to fairly cover the interval $[-1,1]$ during the movement. Each DMP outputs a series of 50 positions that represents a sampling of the trajectory of one joint during the movement. The arm's movement is thus parameterized with 12 weights, represented by the motor space $M=[-1,1]^{12}$.

3) Objects and Tools: Two sticks can be grasped by the handle side (orange side) in order to catch an out-ofreach object. The sticks have length 0.5 and are located at positions $(-0.75,0.25)$ and $(0.75,0.25)$ as in Fig. 2. One stick has a magnet on the end and can catch magnetic objects (represented in blue), and the other stick has a hook-and-loop tape to catch another type of objects (objects represented in green). If the gripper is closed near the handle of one stick (closer than 0.25), this stick is considered grasped and follows the gripper's position and the orientation of the arm's last segment until the gripper opens. In some conditions, we add environmental noise as a gaussian noise of standard deviation 0.1 added to the (normally equal to 0 ) angle between the stick and the arm's last segment, different at each of the 50 movement's steps. If the other side of one stick reaches (within 0.25) a matching object (magnetic or hookand-loop), the object will then follow the end of the stick. Three magnetic objects are located at positions $(-0.3,1.1)$, $(-1.2,1.5)$ and $(-1 ., 1.5)$, so that only one is reachable with the magnetic stick. Three hook-and-loop objects are located at positions $(0.3,1.1),(1 ., 1.5)$ and $(1.2,1.5)$, so that only one is reachable with the hook-and-loop stick. Also, two animals walk randomly following a gaussian noise of standard deviation 0.01 on $X$ and $Y$ dimensions added at each of the 50 steps of a trial. Finally, four static black squares have also no interaction with other objects. The arm, tools and other objects are reset to their initial state at the end of each iteration.

4) Sensory Feedback: At the end of the movement, the robot gets sensory feedback representing the trajectory of the different items of the environment during the arm's movement. This feedback is composed by the position of each item at 3 time points: at steps 17, 33, and 50 during the movement of 50 steps. First, the trajectory of the gripper is represented as a sequence of $X$ and $Y$ positions and aperture $(1$ or -1$)$ of the gripper $\left(S_{\text {Hand }}, 9 \mathrm{D}\right)$. Similarly, the trajectories of the end points of the sticks are sequences of $X$ and $Y$ positions ( $S_{\text {Stick }_{1}}$ and $S_{\text {Stick }_{2}}, 6 \mathrm{D}$ each). Also, the trajectory of each object is a sequence of $X$ and $Y$ positions: $S_{\text {Object }}$ with Object $\in\left\{\right.$ Magnetic $_{1}$, Magnetic Ma $_{2}$, Magnetic ${ }_{3}$, HookLoop 1 , HookLoop 2 , HookLoop 3 , Cat, Dog, Static $_{1}$, Static $_{2}$, Static $_{3}$, Static $\left._{4}\right\}$. Those spaces are all in 6 dimensions $\left([-1.5,1.5]^{6}\right)$. The total sensory space $S$ has 93 dimensions and corresponds to 15 items.

\section{Exploitation Architectures}

An exploitation architecture generates an inverse model of the environment based on a database of previously explored motor commands and their associated sensory feedback. In this paper, we are both interested in the quality of the exploration databases and in comparing the inverse models built by different combinations of exploration database and exploitation architectures. We evaluate the accuracy of the resulting inverse models to reach points in two spaces of interest, $S_{\text {Magnetic } 1}$ and $S_{\text {Hook Loop }}$. Indeed, those spaces represent the only objects that can be moved by one of the sticks as they are not static and not out-of-reach. One set of goals is randomly drawn in the 2D subspace corresponding to the final position of each of the two interesting objects (1000 goals in each).

We define two exploitation architectures generating inverse models: one based on the Nearest Neighbor algorithm (NN, Algo. 7), and one based on the Locally Weighted Linear Regression forward model and an optimization-based inverse model (LWLR, Algo. 8). Given a goal $s_{g}$ (e.g. $s_{g}=(0.5,0.5)$, the final position of the reachable magnetic object), the NN algorithm looks into the explored database, 
finds the nearest sensory reached point $s$ along the dimensions of the target effect space, and returns its associated motor command $m$. On the other hand, the LWLR algorithm builds a forward model based on a locally weighted linear regression, and an optimization algorithm (L-BFGS-B) finds the motor command $m$ that minimizes the distance between the prediction of the sensory feedback and the sensory goal.

\section{RESULTS}

We run 100 trials of 100000 iterations with environmental noise and 100 trials without noise, for each of the 7 exploration architectures (thus 14 conditions). We first measure the total exploration of $6 \mathrm{D}$ spaces of interest $S_{\text {Magnetic }_{1}}$ and $S_{\text {HookLoop }_{1}}$ after 100000 iterations, and provide results depending on the exploration architecture and environmental noise on the orientations of the sticks. Then, for each of the 1400 exploration databases, we test the inverse models generated by the two exploitation architectures in the $2 \mathrm{D}$ subspaces of the final position of the two objects of interests, with the same 1000 random goals for each space. We chose those $2 \mathrm{D}$ spaces as they represent an interesting effect space from the point of view of the experimenter (as in Fig. 3), but the actually learnt skills are higher-dimensional (9D for the hand, $6 \mathrm{D}$ for each tool and object). We provide a measure of competence of each combination of exploration and exploitation architectures as the median reaching error (the median distance between the goals and actually reached sensory points), both when environmental noise was present and when the environment was deterministic.

\section{A. Exploration}

1) Examples of Object Exploration: Figure 3 shows qualitatively the exploration of the two reachable and movable objects (corresponding to sensory spaces $S_{\text {Magnetic }}$ and $S_{\text {HookLoop }_{1}}$ ) for one trial of some exploration architectures, without environmental noise. The blue points are the $2 \mathrm{D}$ end positions of the reachable magnetic object, and green points are the end positions of the reachable hook-and-loop object, for the 100000 iterations of an exploration trial. First, the random motor babbling architecture managed to grab the sticks to move one of the object only for a small proportion of the 100000 iterations. Also, only the modular architectures could explore a large proportion of the 2D spaces.

2) Evolution of Interests in Active Model Babbling: Figure 4 shows one example of the evolution of the interest of some of the 15 modules of exploration architecture MNN-AMB. The first module to make progress is the module learning to move the hand, and its exploration finds the magnetic stick and thus allows the module corresponding to this stick to make more progress (after 10000 iteration), which exploration finally allows the discovery that this stick can be used to move one of the magnetic objects and make progress on that task (after 20000 iteration). Notably, modules corresponding to unreachable or static objects have an interest strictly equal to 0 .
TABLE I

EXPLORATION OF SPACES OF INTEREST

\begin{tabular}{|c|c||c|c|c|c|c|}
\hline $\begin{array}{c}\text { Exploration } \\
\text { architectures }\end{array}$ & $\begin{array}{c}\text { Env. } \\
\text { Noise }\end{array}$ & Min & Q1 & Median & Q3 & Max \\
\hline \hline \multirow{2}{*}{ RmB } & No & 57 & 67 & 73 & 78 & 93 \\
\cline { 2 - 7 } & Yes & 62 & 75 & 80 & 85 & 100 \\
\hline \multirow{2}{*}{ F-NN-RGB } & No & 1 & 1 & 14 & 89 & 380 \\
\cline { 2 - 7 } & Yes & 1 & 1 & 16 & 116 & 746 \\
\hline \multirow{2}{*}{ F-LWLR-RGB } & No & 98 & 203 & 245 & 294 & 442 \\
\cline { 2 - 7 } & Yes & 182 & 319 & 387 & 486 & 818 \\
\hline \multirow{2}{*}{ M-NN-RMB } & No & 285 & 374 & 415 & 456 & 682 \\
\cline { 2 - 7 } & Yes & 356 & 455 & 508 & 563 & 763 \\
\hline \multirow{2}{*}{ M-NN-AMB } & No & 88 & 452 & 536 & 668 & 1380 \\
\cline { 2 - 7 } & Yes & 156 & 431 & 517 & 721 & 1453 \\
\hline \multirow{2}{*}{ M-LWLR-RMB } & No & 368 & 512 & 555 & 607 & 801 \\
\cline { 2 - 7 } & Yes & 449 & 574 & 623 & 691 & 906 \\
\hline \multirow{2}{*}{ M-LWLR-AMB } & No & 456 & 743 & 870 & 1046 & 1440 \\
\cline { 2 - 7 } & Yes & 522 & 811 & 987 & 1153 & 1752 \\
\hline
\end{tabular}

3) Exploration Measure: The total exploration is measured in $S_{\text {Magnetic }_{1}}$ and $S_{\text {HookLoop }_{1}}$ as the number of cells reached in a discretized grid of $10^{6}$ cells (10 cells on each of the 6 dimensions). For each exploration architecture, we provide in Table I the median, extrema and quartiles of the number of reached cells (median on 2 spaces times 100 trials). In the following, we give results of non-parametric statistical Mann-Whitney $U$ tests for pairs of conditions.

First of all, the comparison of any of the flat exploration architectures (using NN or LWLR, with or without environmental noise) with any of the modular exploration architectures shows that flat architectures have explored less than modular architectures $(p<0.05)$. The effect is small for example if we compare condition F-LWLR-RGB with environmental noise (median 387 reached cells) with condition M-NN-RMB without noise (median 415). However, the difference is large between this flat architecture and the best exploring modular architecture, M-LWLR-AMB with environmental noise (median 987).

Secondly, the comparison of the conditions where only the model babbling choice differs shows that without environmental noise, active model babbling increases exploration with respect to random model babbling. Indeed, architecture M-NN-RMB has explored less (median 415) than architecture M-NN-AMB (median 536, $p<10^{-23}$ ), and architecture M-LWLR-RMB also has explored less (median 555) than architecture M-LWLR-AMB (median $870, p<10^{-55}$ ). If we consider environmental noise, the random model babbling architecture using LWLR (median 623) has explored less than the active one (median 987, $p<10^{-39}$ ).

\section{B. Exploitation}

The quality of the different inverse models is assessed at the end of the 100000 exploration iterations, by giving random goals in $S_{\text {Magnetic }}$ and $S_{\text {Hook Loop }_{1}}$ and measuring the distance between goals and reached sensory points (without environmental noise). We draw 1000 random sensory goals in each of two spaces of interest, $S_{\text {Magnetic }}$ and $S_{\text {HookLoop }_{1}}$, and use those same goals for the evaluation of each com- 


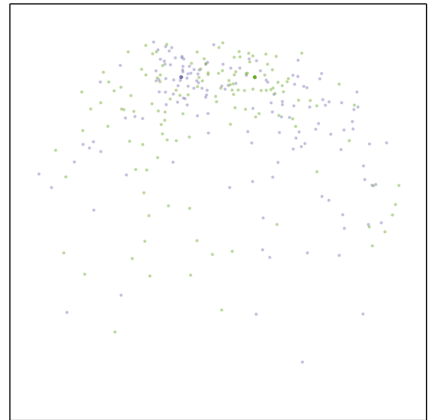

(a) $\mathrm{RmB}$

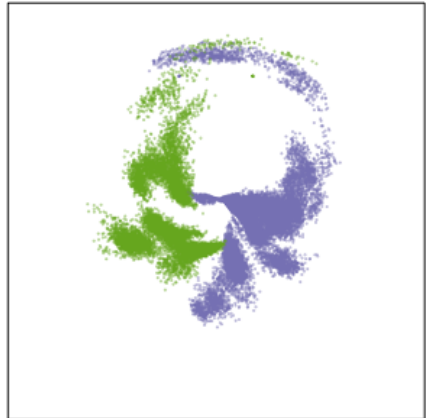

(b) F-NN-RGB

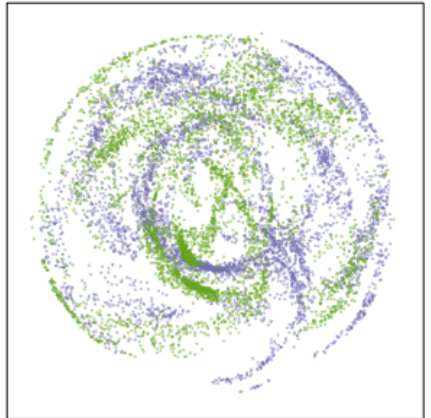

(c) M-NN-RMB

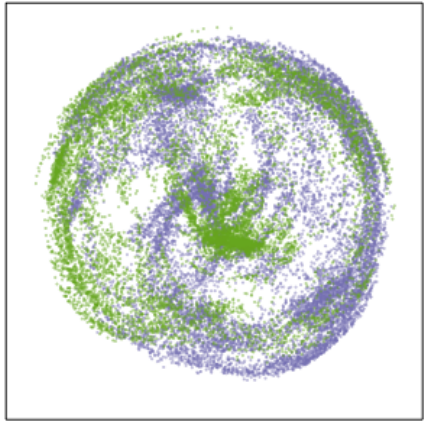

(d) M-NN-AMB

Fig. 3. Position of the two reachable and movable objects at the end of each of the 100000 iterations, for one trial of some exploration architecture. Blue points: position of reachable magnetic object. Green points: reachable hook-and-loop object.

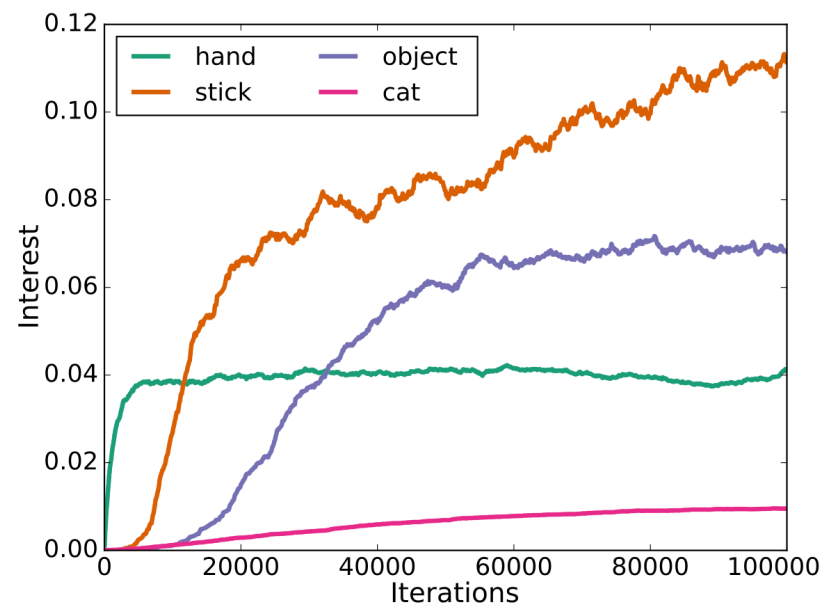

Fig. 4. Interest of modules along the 100000 iterations, with exploration architecture M-NN-AMB. We show the interest of modules exploring the spaces of the hand, magnetic stick, reachable magnetic object and the cat.

bination of exploration and exploitation architectures. Table III provides the median distance between goals and reached sensory points for each condition (for 2000 points times 100 trials). In the following, we give results of non-parametric statistical Mann-Whitney U tests for pairs of conditions.

Firstly, both if we consider conditions with environmental noise or not, all databases generated by flat exploration architectures and tested by any of the two exploitation architectures show a larger competence error than any of the databases explored with modular architectures and tested with both exploitation architecture $\left(p<10^{-100}\right)$. For instance, without environmental noise, the best performing flat condition is F-LWLR-RGB exploited with the NN algorithm, with a median competence error of 0.123 , whereas the worst performing modular condition is M-NN-RMB, exploited with the LWLR algorithm, with an error of 0.050 .

Secondly, considering only exploration conditions without environmental noise, all databases generated with RMB architectures and tested with any of the two exploitation architectures show a larger competence error than any of the databases generated with AMB and tested with both exploitation architectures $(p<0.05)$. For instance, the median competence error using RMB and the $\mathrm{NN}$ algorithm both in
TABLE II

COMPETENCE ERROR IN SPACES OF INTEREST

\begin{tabular}{|c|c||c|c|}
\hline $\begin{array}{c}\text { Exploration } \\
\text { architecture }\end{array}$ & $\begin{array}{c}\text { Env. } \\
\text { Noise }\end{array}$ & NN & LWLR \\
\hline \hline \multirow{2}{*}{ RmB } & No & 0.185 & 0.711 \\
\cline { 2 - 4 } & Yes & 0.307 & 0.871 \\
\hline \multirow{2}{*}{ F-NN-RGB } & No & 0.745 & 1.018 \\
\cline { 2 - 4 } & Yes & 1.174 & 1.253 \\
\hline \multirow{2}{*}{ F-LWLR-RGB } & No & 0.123 & 0.171 \\
\cline { 2 - 4 } & Yes & 0.376 & 0.422 \\
\hline \multirow{2}{*}{ M-NN-RMB } & No & 0.046 & 0.050 \\
\cline { 2 - 4 } & Yes & 0.248 & 0.261 \\
\hline \multirow{2}{*}{ M-NN-AMB } & No & 0.035 & 0.037 \\
\cline { 2 - 4 } & Yes & 0.285 & 0.300 \\
\hline \multirow{2}{*}{ M-LWLR-RMB } & No & 0.038 & 0.039 \\
\cline { 2 - 4 } & Yes & 0.216 & 0.227 \\
\hline \multirow{2}{*}{ M-LWLR-AMB } & No & 0.026 & 0.026 \\
\cline { 2 - 4 } & Yes & 0.215 & 0.226 \\
\hline
\end{tabular}

exploration and exploitation is 0.046 whereas with AMB it is 0.035. Using LWLR, those errors are 0.039 and 0.026 .

\section{DISCUSSION}

We have introduced two new algorithmic architectures for incremental exploration of sensorimotor spaces, exploiting a modular representation of these spaces. Random Model Babbling selects randomly which model to explore (which is itself explored through goal babbling) and Active Model Babbling (MACOB) uses a multi-armed bandit algorithm to maximize empirical learning progress. In a simulation involving structured continuous high-dimensional motor (12D) and sensory (93D) spaces, we showed that these modular architectures were vastly more efficient than goal babbling methods used with flat representations, for all combinations of inverse models in the exploration and exploitation architectures. In particular, by focusing exploration on relevant parts of the space, modular architectures allowed the learner to discover efficiently how to move various objects using various tools, while flat architectures were not able to discover large parts of the space of effects. We also showed that active model babbling was significantly more efficient than random model babbling, yet the difference was smaller than between modular and flat architectures. 
To extend the scope of these results, further work is needed to study other experimental setups involving real physical robots, tools and objects, and setups with more motor and sensory dimensions. Also, in our experiment, the objects are reset to their initial position at each iteration. In a setup where we reset the object to a random position [27], we showed that similar agents could transfer the knowledge about tool manipulation to reach the object in one position in order to bootstrap exploration to reach the object in a new position.

Given the potential importance of modular representations to address the challenge of incremental learning of skills in high-dimensional spaces, and within a life-long developmental perspective, this work also points to the need for algorithmic mechanisms that can generate automatically such representations. However, from a cognitive point of view regarding human tool use learning, it is reasonable to suppose that the brain has sufficient knowledge about the concept of objects and their properties at the time of understanding object interactions and tool use, and it makes sense to build upon those representations in order to model tool use learning [28]. The modular representation we give to the learning agent thus seems natural as each sensory subspace corresponds to the behavior of one object in the scene.

Finally, also from the perspective of modeling tool use discovery, an interesting feature of our setup was that "tool" objects did not have a special status: they were represented in the same way as any other object. Yet, the active model babbling architecture converged to explore preferentially these objects and discovered their actual use as tools. In [29], the learning agents had a hierarchical organization of modules which was imposed and not built autonomously. Further algorithms could be developed to transform the modular architecture into a hierarchical one where causal dependencies between objects could be represented and leveraged, with the discovery of explicit object categories such as "tools". A possible approach could be to differentiate "tools" using a relative measure of learning progress, following the approach presented in [30, sec. VIII. B. 2] to differentiate the self/body, physical objects and "others".

\section{REFERENCES}

[1] A. Cangelosi, M. Schlesinger, and L. B. Smith, Developmental robotics: From babies to robots. MIT Press, 2015.

[2] G. Baldassarre and M. Mirolli, Intrinsically Motivated Learning in Natural and Artificial Systems. Springer, 2013.

[3] E. Ugur, Y. Nagai, E. Sahin, and E. Oztop, "Staged development of robot skills: Behavior formation, affordance learning and imitation with motionese," IEEE Transactions on Autonomous Mental Development, vol. 7, no. 2, 2015.

[4] F. Stulp, G. Raiola, A. Hoarau, S. Ivaldi, and O. Sigaud, "Learning compact parameterized skills with a single regression," in IEEE-RAS International Conference on Humanoid Robots, 2013.

[5] B. Da Silva, G. Konidaris, and A. Barto, "Learning parameterized skills," in ICML, 2012, pp. 1679-1686.

[6] A. Ude, A. Gams, T. Asfour, and J. Morimoto, "Task-specific generalization of discrete and periodic dynamic movement primitives," Robotics, IEEE Transactions on, vol. 26, no. 5, pp. 800-815, 2010.

[7] A. Baranes and P.-Y. Oudeyer, "Active learning of inverse models with intrinsically motivated goal exploration in robots," Robotics and Autonomous Systems, vol. 61, no. 1, 2013.
[8] A. G. Kupcsik, M. P. Deisenroth, J. Peters, and G. Neumann, "Dataefficient generalization of robot skills with contextual policy search." in $A A A I, 2013$.

[9] M. Rolf, J. Steil, and M. Gienger, "Goal babbling permits direct learning of inverse kinematics," Autonomous Mental Development, IEEE Transactions on, vol. 2, no. 3, 2010.

[10] A. Fabisch and J. H. Metzen, "Active contextual policy search," The Journal of Machine Learning Research, vol. 15, no. 1, pp. 3371-3399, 2014.

[11] F. Stulp and O. Sigaud, "Robot skill learning: From reinforcement learning to evolution strategies," Paladyn. Journal of Behavioral Robotics, vol. 4, no. 1, pp. 49-61, September 2013.

[12] F. Stulp, L. Herlant, A. Hoarau, and G. Raiola, "Simultaneous on-line discovery and improvement of robotic skill options," in International Conference on Intelligent Robots and Systems (IROS), 2014.

[13] C. Moulin-Frier, S. M. Nguyen, and P.-Y. Oudeyer, "Self-organization of early vocal development in infants and machines: the role of intrinsic motivation," Frontiers in Psychology, vol. 4, 2014.

[14] S. M. Nguyen and P.-Y. Oudeyer, "Socially guided intrinsic motivation for robot learning of motor skills," Autonomous Robots, vol. 36, no. 3, pp. 273-294, 2014.

[15] N. Chentanez, A. G. Barto, and S. P. Singh, "Intrinsically motivated reinforcement learning," in Advances in Neural Information Processing Systems 17, L. K. Saul, Y. Weiss, and L. Bottou, Eds. MIT Press, 2005, pp. 1281-1288.

[16] J. Schmidhuber, "Formal theory of creativity, fun, and intrinsic motivation (1990-2010)," Autonomous Mental Development, IEEE Transactions on, vol. 2, no. 3, 2010.

[17] F. Guerin, N. Kruger, and D. Kraft, "A survey of the ontogeny of tool use: from sensorimotor experience to planning," Autonomous Mental Development, IEEE Transactions on, vol. 5, no. 1, 2013.

[18] A. Stoytchev, "Behavior-grounded representation of tool affordances," in Proceedings of the 2005 IEEE International Conference on Robotics and Automation. IEEE, 2005.

[19] A. Antunes, G. Saponaro, A. Dehban, L. Jamone, R. Ventura, A. Bernardino, and J. Santos-Victor, "Robotic tool use and problem solving based on probabilistic planning and learned affordances," in IROS 2015 Workshop on Learning Object Affordances: a fundamental step to allow prediction, planning and tool use?

[20] V. Tikhanoff, U. Pattacini, L. Natale, and G. Metta, "Exploring affordances and tool use on the icub," in 13th IEEE-RAS International Conference on Humanoid Robots (Humanoids), 2013.

[21] C. Moulin-Frier, P. Rouanet, P.-Y. Oudeyer, and others, "Explauto: an open-source Python library to study autonomous exploration in developmental robotics," in ICDL-Epirob-International Conference on Development and Learning, Epirob, 2014.

[22] C. Moulin-Frier and P.-Y. Oudeyer, "Exploration strategies in developmental robotics: a unified probabilistic framework," in ICDL-Epirob - International Conference on Development and Learning, Epirob, Osaka, Japan, 2013.

[23] S. Nguyen and P.-Y. Oudeyer, "Active choice of teachers, learning strategies and goals for a socially guided intrinsic motivation learner," Paladyn, vol. 3, no. 3, 2012.

[24] W. S. Cleveland and S. J. Devlin, "Locally weighted regression: an approach to regression analysis by local fitting," Journal of the American statistical association, vol. 83, no. 403, pp. 596-610, 1988.

[25] R. H. Byrd, P. Lu, J. Nocedal, and C. Zhu, "A limited memory algorithm for bound constrained optimization," SIAM Journal on Scientific Computing, vol. 16, no. 5, pp. 1190-1208, 1995.

[26] A. J. Ijspeert, J. Nakanishi, H. Hoffmann, P. Pastor, and S. Schaal, "Dynamical movement primitives: learning attractor models for motor behaviors," Neural computation, vol. 25, no. 2, 2013.

[27] S. Forestier and P.-Y. Oudeyer, "Overlapping waves in tool use development: a curiosity-driven computational model," in Sixth Joint IEEE International Conference on Development and Learning and Epigenetic Robotics (ICDL-EpiRob), 2016.

[28] B. M. Lake, T. D. Ullman, J. B. Tenenbaum, and S. J. Gershman, "Building machines that learn and think like people," CoRR, vol. abs/1604.00289, 2016.

[29] S. Forestier and P.-Y. Oudeyer, "Curiosity-driven development of tool use precursors: a computational model," in Proceedings of the 38th Annual Meeting of the Cognitive Science Society, 2016.

[30] P.-Y. Oudeyer, F. Kaplan, and V. V. Hafner, "Intrinsic Motivation Systems for Autonomous Mental Development," IEEE Transactions on Evolutionary Computation, vol. 11, no. 2, Apr. 2007. 